\title{
Hierarchical Dynamic Loop Self-Scheduling on Distributed-Memory Systems Using an MPI+MPI Approach
}

\author{
Ahmed Eleliemy and Florina M. Ciorba \\ Department of Mathematics and Computer Science \\ University of Basel, Switzerland \\ \{firstname.lastname\}@unibas.ch
}




\section{Contents}

1 Introduction 4

2 Background and Related Work 6

3 The Hierarchical DLS Approach 9

4 Experimental Setup 11

5 Experimental Evaluation 13

6 Conclusion and Future Work 17 


\begin{abstract}
Computationally-intensive loops are the primary source of parallelism in scientific applications. Such loops are often irregular and a balanced execution of their loop iterations is critical for achieving high performance. However, several factors may lead to an imbalanced load execution, such as problem characteristics, algorithmic, and systemic variations. Dynamic loop self-scheduling (DLS) techniques are devised to mitigate these factors, and consequently, improve application performance. On distributed-memory systems, DLS techniques can be implemented using a hierarchical master-worker execution model and are, therefore, called hierarchical DLS techniques. These techniques self-schedule loop iterations at two levels of hardware parallelism: across and within compute nodes. Hybrid programming approaches that combine the message passing interface (MPI) with open multi-processing (OpenMP) dominate the implementation of hierarchical DLS techniques. The MPI-3 standard includes the feature of sharing memory regions among MPI processes. This feature introduced the MPI+MPI approach that simplifies the implementation of parallel scientific applications. The present work designs and implements hierarchical DLS techniques by exploiting the MPI+MPI approach. Four well-known DLS techniques are considered in the evaluation proposed herein. The results indicate certain performance advantages of the proposed approach compared to the hybrid MPI+OpenMP approach.
\end{abstract}

Keywords: Dynamic loop self-scheduling, Hierarchical dynamic loop self-scheduling, MPI, OpenMP, MPI+OpenMP, MPI+MPI 


\section{Introduction}

Today, multi- and many-core processors are at the basis of modern large-scale distributed-memory systems. The message passing interface (MPI) [1] is the most dominant programming approach for scientific applications that execute on large-scale distributed-memory systems. MPI provides portable performance on most of the modern distributed-memory systems. However, the increasing number of computing cores within a single processor creates specific performance challenges for MPI. For instance, mapping MPI processes to individual cores results in limited sub-problem sizes per MPI process; the memory capacity per core does not grow as fast as the growth of the number of cores within a processor 22. Also, exchanging data between two MPI processes that execute on the same processor may pose another performance challenge; this data exchange stresses the memory subsystems by performing memory-to-memory copy operations which may degrade applications performance [3]. These challenges motivated the intensive use of hybrid parallel programming in scientific applications. The hybrid programming approaches combine distributed-memory and shared-memory programming approaches. For instance, the combination of MPI with OpenMP [4], known as MPI+OpenMP, is widely used in several scientific applications 5 7]. However, exploiting two parallel programming models and managing parallelism by two different runtime systems within the same application may lead to complex applications codes. The code complexity may pose performance and maintainability challenges.

The MPI forum added, in the MPI-3 standard, the feature of sharing memory regions between MPI processes that reside on a shared-memory system 1]. This introduced a new parallel programming approach called MPI+MPI approach 2. The new approach can be seen as a technological advancement that may result in enhancing applications' performance and reducing applications' code complexity. The present work focuses on exploiting the MPI+MPI approach in designing and implementing efficient hierarchical dynamic loop selfscheduling (DLS) techniques.

Executing scientific applications on multi-processor systems requires all processing elements to reach nearly equal finishing times. DLS techniques aim to balance loop iterations' execution across all processing elements by mitigating the effects of several factors, such as problem characteristic, algorithmic and systemic variations, which may hinder such a balanced execution 8, 9].

DLS techniques typically employ a master-worker execution model $10-12$. This model includes a processing entity called master that is responsible for calculating and assigning chunks of loop iterations to all the other entities (workers). Exploiting this model has scaling [12], and therefore, the DLS techniques have evolved to employ a hierarchical master-worker execution model [13]. This model includes two levels of masters: global and local masters. The global master calculates and assigns chunks to local masters, each of them being responsible for calculating and assigning sub-chunks to its group of workers. The global master and local masters may exploit different DLS techniques. The use of DLS techniques at two levels is also referred to as a hierarchical DLS 
technique.

Hybrid MPI+OpenMP is a common programming approach to implement hierarchical DLS techniques. However, it has specific performance challenges, such as, the added overhead for the management of two levels of parallelism using two different runtime systems.

The OpenMP threads (workers) require synchronization before requesting and executing chunks, i.e., only the main thread is allowed to call MPI communication functions, such as MPI_Send and MPI_Receive [14. Otherwise, a complex implementation is needed to allow individual OpenMP threads to perform MPI calls.

This work proposes a novel approach for designing and developing hierarchical DLS techniques for distributed-memory systems. It extends the distributed chunk calculation approach 15 by allowing any group of workers to reside on a shared-memory system to form a shared work queue where the chunks to be executed by this group are stored. The novelty of the proposed approach lies in the fact that the responsibility of the work queue is shared among the workers of the group. The present work considers the shared-memory features offered in the MPI-3 standard to assign chunks to individual MPI processes in two stages. In the first stage, the fastest MPI process within a compute node obtains a chunk based on a selected DLS technique. The fastest MPI process uses the obtained chunk to fill the local shared queue. In the second stage, the MPI processes within the same shared-memory system use a different or a similar DLS technique to obtain sub-chucks from the shared local work queue.

The importance of this work is enabling efficient and scalable implementations of hierarchical DLS techniques using a single programming model which promotes code clarity, maintainability, and decreased effort in debugging work. Moreover, it preserves the research efforts spent in developing DLS techniques using MPI. The main contributions of this work are as follows. (1) Proposal and implementation of a hierarchical version of DLS techniques for distributed-memory systems using the MPI+MPI approach. (2) Evaluation of using the MPI+MPI approach in developing hierarchical DLS techniques.

The remainder of this work is organized as follows. Section 2 provides the necessary information to understand the DLS techniques, and presents similar research efforts reported in the literature. The proposed approach is described in Section 3. The experimental design and the evaluation results are discussed in Sections 4 and 5 , respectively. The conclusions and the future work are outlined in Section 6 . 


\section{Background and Related Work}

Load Balancing: The efficient execution of parallel applications requires balancing the workload among all processing elements, which means that all workers should finish almost at the same time. In practice, such efficient executions are challenging to be achieved. Many factors, such as problem characteristics, algorithmic, and systemic variations hinder them. Several load balancing techniques have been introduced in the literature $16-21$ to fit the needs of different applications.

Loop Scheduling Techniques: In scientific applications, loops are the main source of parallelism 22]. Loop scheduling techniques have been introduced to achieve a balanced load execution of loop iterations. When loops have no cross-iteration dependencies, loop scheduling techniques map individual loop iterations to different processing elements aiming to have nearly equal finish times on all processing elements. Loop scheduling techniques can be categorized into static and dynamic loop scheduling. The time when scheduling decisions are taken is the key difference between both categories. Static loop scheduling (SLS) techniques take scheduling decisions prior to applications' execution, while dynamic loop scheduling (DLS) techniques take scheduling decisions during applications' execution. Therefore, SLS techniques have less scheduling overhead than DLS, and DLS techniques can achieve better load balanced executions than SLS in highly dynamic execution environments.

DLS techniques can further be divided into non-adaptive and adaptive techniques. The non-adaptive techniques utilize certain information that is obtained prior to the application execution. The adaptive techniques regularly obtain information during the application execution, and the scheduling decisions are taken based on that new information. The adaptive techniques incur a significant scheduling overhead compared to non-adaptive techniques, and outperform the non-adaptive ones in highly irregular execution environments.

Selected DLS Techniques: In addition to the extreme cases of loop scheduling techniques: fully-static (STATIC) and fully-dynamic (SS), we selected three DLS techniques: guided self-scheduling (GSS) [23, trapezoid self-scheduling (TSS) [24, and Factoring (FAC) 25]. These techniques are remarkable points on the DLS spectrum, since they have competitive performance in different applications, and they are at the basis of other DLS techniques, such as weighted factoring [26, adaptive weighted factoring (AWF) 8], and trapezoid factoring self-scheduling (TFSS) 27.

In STATIC, each processing element obtains a chunk of $N / P$, where $N$ and $P$ are the total number of iterations and the total number of processing elements, respectively. STATIC incurs the lowest scheduling overhead.

SS 28 is a dynamic self-scheduling technique where the chunk size is always one iteration. SS has the highest scheduling overhead. However, SS can achieve a highly load-balanced execution in highly irregular execution environments.

In GSS [23] at every scheduling step, the remaining loop iterations are divided by the total number of processing elements. GSS is a compromise between the maximum load balancing that can be achieved using SS and the lowest 
scheduling overhead that is incurred in STATIC.

Unlike GSS, TSS 24 uses a linear function to decrement chunk sizes. This linearity results in low scheduling overhead in each scheduling step compared to GSS.

FAC 25] is another DLS technique that schedules the loop iterations in batches of equally-sized chunks. FAC evolved from comprehensive probabilistic analyses, and assumes prior knowledge about the standard deviation of loop iterations execution times $\sigma$ and their mean execution time $\mu$. Another practical implementation of FAC, denoted FAC2, assigns half of the remaining loop iterations for every batch. The initial chunk size of FAC2 is half of the initial chunk size of GSS. If more time-consuming loop iterations are at the beginning of the loop, FAC2 may balance their execution better than GSS.

Related Work: Most of the DLS techniques were introduced between the late of 1980s and the early of 2000s 8, 14, 23 26, 28 30. The DLS techniques target loop executions on both, shared-memory and distributed-memory systems. Therefore, their implementation strategies and technologies evolved to fit both system types.

On shared-memory systems, DLS techniques have been implemented using multi-threading. Threads share certain variables (the last scheduled loop index and the remaining loop iterations) that control the loop execution, and each thread calculates its own chunk size based on the values of these variables. Atomic operations or locks are used to keep the shared variables up to date. Open multi-processing (OpenMP) is considered as one of the most dominant multi-threading APIs for shared-memory systems. The OpenMP-5 standard [4] uses the schedule clause to define three different loop scheduling options: static, dynamic, and guided. Table 1 shows the mapping between the OpenMP schedule clause and the DLS techniques discussed earlier in this section. The OpenMP standard uses the schedule(runtime) clause to allow users to select a certain scheduling technique during the execution of the application. A recent research effort implemented other DLS techniques, namely TSS, FAC2, $\mathrm{WF}$, and random self-scheduling in an open source OpenMP runtime library, called LaPeSD-libGOMP 31. The authors exploited the schedule(runtime) clause together with certain user-defined environment variables to select one of the DLS techniques that they implemented in the LaPeSD-libGOMP runtime.

On distributed-memory systems, the dominant approach for developing DLS

Table 1: Mapping between the DLS techniques and the OpenMP schedule clause options

\begin{tabular}{l|l} 
DLS technique & OpenMP schedule clause \\
\hline STATIC & schedule(static) \\
SS & schedule(dynamic,1) \\
GSS & schedule(guided,1)
\end{tabular}

${ }^{1}$ https://github.com/lapesd/libgomp 
techniques is the message-passing interface (MPI) 1]. The MPI-based implementation requires employing a master-worker execution model, where a certain MPI process, called master is responsible for calculating and assigning chunks to other free and requesting MPI processes, called workers.

A load balancing tool, called DLB_tool that integrates several DLS techniques was introduced 10 . The DLB_tool employed the master-worker execution model implemented using the classical two-sided MPI communication. The tool assumed a non-dedicated master process that participates in the loop execution similar to the workers.

An enhanced tool for dynamic load balancing, called dynamic load balancing library (DLBL) [11] was the first to utilize MPI one-sided communication. In DLBL, the master process receives work requests, and for each request, it calculates the size of the new chunk. The master process calls a handler function on the worker side. Workers obtain the data of the new chunks from the master process.

In the master-worker execution model, the number of requests that could be received by the master process is proportional to the total number of workers. For a large number of workers, the master may simultaneously receive a large number work requests, and if the handling og the work requests is inefficient, the master becomes a performance bottleneck. To overcome such limitation, certain research efforts proposed the use of the hierarchical master-worker approach. For instance, a distributed self-scheduling scheme (HDSS) using the hierarchical master-worker model was introduced 13. Unlike the LB-tool, HDSS dedicated the master process for handling the worker requests. The proposed scheme was implemented using MPI and its classical two-sided communication.

Another research effort discussed the adverse impact of the foreman-worker (master-worker) model 12 on DLS techniques. The authors suggested a new execution model using processor groups. The idea was to form a few groups of processors, where each group executes a specified portion of the iteration space using the master-worker model. However, the master process of each group has to periodically update a global master process, called manager, with the ratio of the remaining iterations and the available workers. When the reported ratio exceeds a certain threshold, the manager migrates workers between processor groups. This research effort is similar to the present work as both balance the loop execution using two levels of scheduling. However, the present work differs by exploiting different DLS techniques at the first level while the suggested execution model in [12] statically divides the loop iteration space among processor groups. Moreover, the present work avoids worker migration that may result in performance degradation when the cost of managing and serving requests from the migrated workers become relatively large.

Recently, a distributed chunk-calculation approach was proposed for developing DLS techniques executing on distributed-memory systems 15]. This approach eliminated the use of the master-worker model by exploiting the one-sided communication features offered in the MPI-3 standard. The distributed chunk-calculation approach uses formulas of DLS techniques that depend on a single value, namely, the latest scheduling step. The main idea is 
that each worker can atomically get and increment the latest scheduling step, then workers can simultaneously and independently compute their own chunk sizes. This avoids the need of having a master entity that sequentially assigns and computes chunks to requesting workers.

Modern high performance computing (HPC) systems are clusters of multiand many-core systems connected via high-speed interconnection networks 7 . Developers often combine two different programming models to target such systems. A common approach is to use MPI [1] for inter-node communication and OpenMP [4] for programming the shared-memory systems 6]. In the context of DLS techniques, the hierarchical loop scheduling (HLS) [14] was one of the earliest efforts to use MPI+OpenMP programming model. In HLS, a free worker (MPI process) requests a chunk from the master rank which calculates and assigns the chunk based on a certain performance function [30]. The workers (MPI processes) locally use OpenMP loop scheduling techniques, such as static, dynamic, and guided to execute the assigned chunk.

The current work addresses the following limitations of the HLS approach: (1) the implicit synchronization between all OpenMP threads at the end of the execution of each chunk and (2) the limited choice of DLS techniques currently specified in the OpenMP standard.

\section{The Hierarchical DLS Approach}

The proposed approach applies two DLS techniques at the intra- and inter-node levels as follows: one MPI process creates a global shared-memory region, called global work queue. This global queue stores information regarding the latest scheduling step and the total scheduled loop iterations [15]. Using MPI_Win_allocate_shared, the MPI processes within one compute node create another shared-memory region, called local work queue. This local queue stores information regarding the latest scheduling step and the total scheduled loop iterations by the MPI processes within that physical node. Whenever an MPI process becomes free, it obtains a sub-chunk from the local work queue. If there are no sub-chunks, the MPI process tries to obtain a chunk from the global work queue, and fills the empty local work queue. In the proposed hierarchical DLS approach, the MPI processes do not wait for each other to fill the local queue. The responsibility of obtaining work is not assigned to a specific MPI process, as the fastest MPI process always takes this responsibility. Figure 1 illustrates the proposed hierarchical DLS approach and its MPI+MPI-based approach.

Unlike existing MPI+OpenMP implementations of DLS techniques, the proposed approach avoids the implicit synchronization that is required at the end of executing the chunks. Figure 2 illustrates the undesired implicit thread synchronization when using the MPI+OpenMP approach. OpenMP Thread 1 finished its sub-chunk earlier than the rest of the threads. However, it has to wait for the slowest OpenMP Thread (OpenMP Thread 7). At the second chunk, the same scenario was repeated when OpenMP Threads 6 and 7 finished earlier than the 


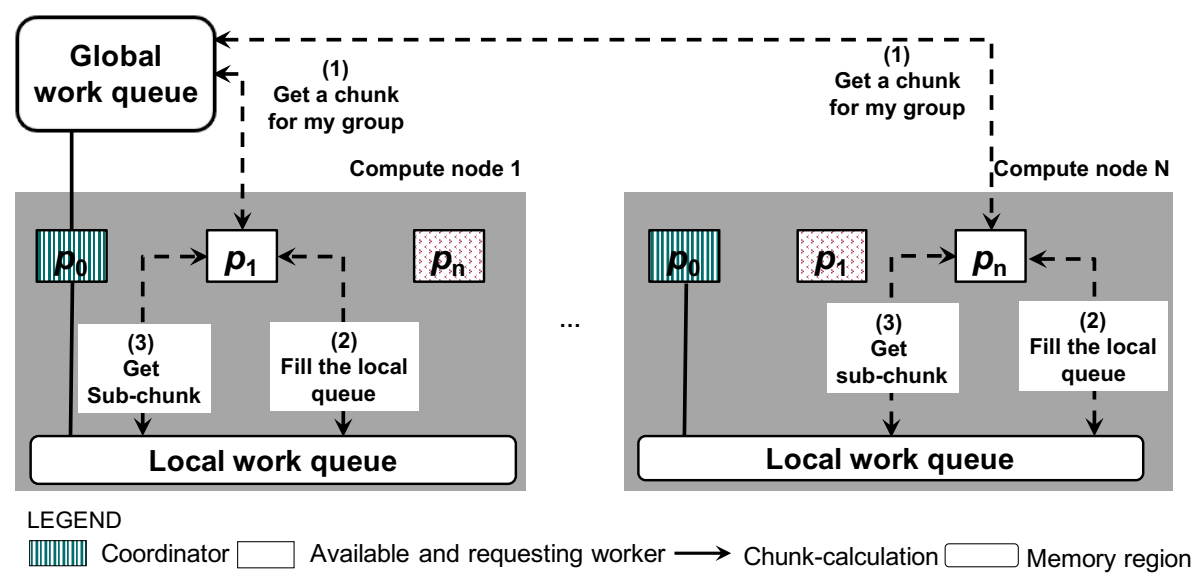

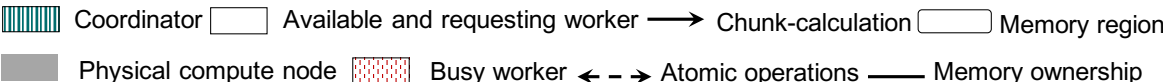

Figure 1: Illustration of the proposed hierarchical DLS techniques using MPI+MPI-based approach.

rest.

Figure 3 shows the desired optimal execution scenario at the shared-memory level. Worker 1 finished earlier than the rest, however, it immediately obtained a new chunk to fill the local queue, and then it obtained a sub-chunk for itself. Once any other worker finished its sub-chunk, it could directly obtain a sub-chunk from the most recent chunk obtained by Worker 1. In Figure 3 , the parallel time to execute the loop $t_{\text {end }}^{\prime}$ is less than $t_{\text {end }}$ in Figure 2.

Compared to the proposed approach, one could state that the main issue of the MPI+OpenMP approach is the implicit barrier at the end of executing each chunk of a loop iteration. Such an issue could be solved by using the nowait

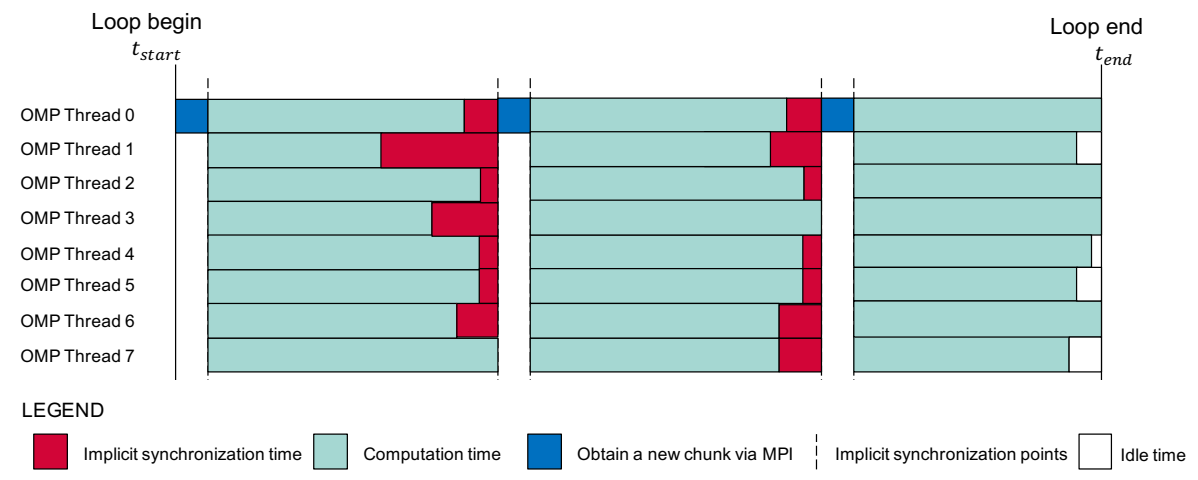

Figure 2: Illustration of the undesired synchronization with the MPI+OpenMP approach at the shared-memory level. 
clause that allows OpenMP threads to continue their execution when there are no more loop iterations to execute. However, the use of the nowait clause requires all OpenMP threads to initiate MPI_Send and MPI_Recv calls, i.e., the fastest OpenMP thread may differ from one chunk to another. Therefore, the implementation would require many synchronization statements to guarantee the exclusive request of new chunks for only one thread at a time. This leads to more complicated codes, which are hard to tune and maintain.

Another significant advantage of the MPI+OpenMP approach is the flexible selection of different loop scheduling technique; i.e., one may specify the scheduling technique using the schedule clause [31]. The MPI-based libraries that implement DLS techniques, such as the DLB_tool [11, often guarantee the same flexibility, e.g., one input parameter specifies the selected DLS technique. The present work discusses and evaluates the advantages and disadvantages of the proposed approach. However, introducing a library that uses the proposed approach and provides the same flexibility is feasible and planned as future work.

\section{Experimental Setup}

Selected Applications: Two scientific applications are used to assess and compare the performance of the proposed MPI+MPI hierarchical DLS approach: PSIA 32,33 and Mandelbrot 34. PSIA is a parallel version of the spin-image algorithm (SIA), which converts a 3D object into a set of 2D images [35]. The generated spin-images can be used as a shape descriptor. The efficient generation of spin-images has a high-interest for 3D object recognition and categorization. Dynamic loop scheduling techniques have been used to implement an efficient parallel spin-image generation algorithm [36. Therefore, the present work examines the potential of using hierarchical DLS techniques in enhancing the performance of PSIA.

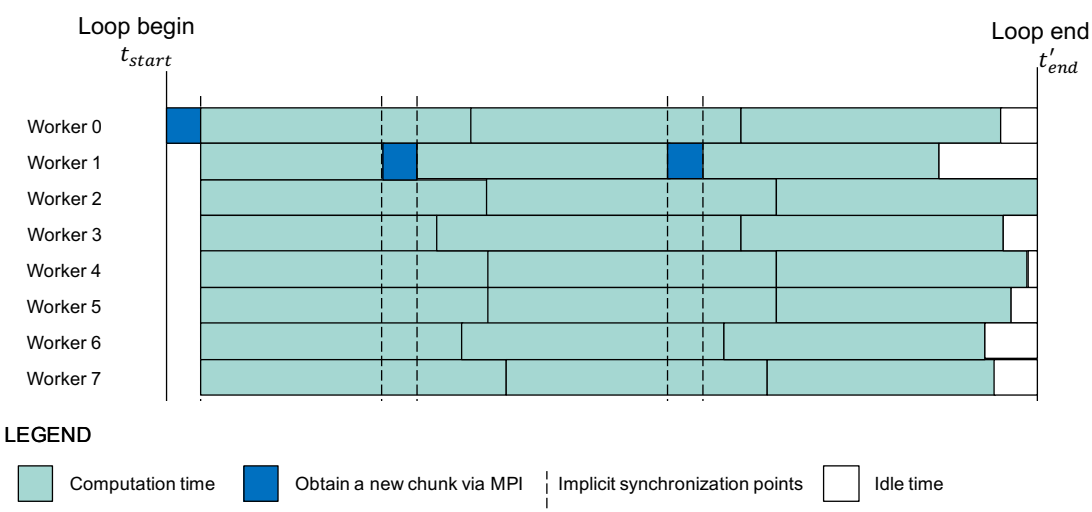

Figure 3: Illustration of an ideal execution scenario at the shared-memory level. 
Mandelbrot is a parallel application that calculates the Mandelbrot set 34 . Mandelbrot is selected as a test application due to high algorithmic load imbalance that motivated its use as a kernel for DLS performance evaluation in the literature 13, 14,30. Both applications are computationally-intensive and contain a single large parallel loop that dominates the application execution time.

Implementation Approaches: Hierarchical DLS techniques can be implemented either using the hierarchical master-worker 13 or using the distributed chunk-calculation model [15. The present work evaluates the use of two different implementations, MPI+OpenMP and MPI+MPI, to complement the distributed chunk-calculation approach (see Section 2).

The MPI+OpenMP implementation complements the distributed chunk-calculation approach by the use of OpenMP at the shared-memory level. It maps one MPI process per each compute node. The mapped MPI processes communicate and cooperate to obtain chunks using one of the following DLS techniques: STATIC, SS, GSS, TSS, and FAC2. Every MPI process uses the OpenMP runtime to create a number of threads equal to the number of its computing cores. The threads use the OpenMP loop scheduling techniques (static, dynamic, and guided) to execute the chunks obtained from their (owner) MPI process. The MPI+MPI implementation complements the distributed-chunk calculation approach by the use of MPI shared-memory capabilities as explained in Section 3, i.e., it forms shared local queues at the compute node level (see Figure 1).

Selected System: The target hardware is a small HPC cluster (called miniHPC [37) that consists of 26 compute nodes. The first 22 nodes are two-socket Intel Xeon E5-2640 processors with 20 cores, 64 GB RAM, and $2.4 \mathrm{GHz} \mathrm{CPU}$ frequency. The remaining 4 nodes are standalone Intel Xeon Phi 7210 manycore processors with 64 cores and 96 GB RAM. All nodes are interconnected using Intel Omni-Path fabric in a non-blocking fat-tree topology. The network bandwidth and latency are $100 \mathrm{GBit} / \mathrm{s}$ and $100 \mathrm{~ns}$, respectively. The miniHPC cluster is used for educational and research purposes. Therefore, sixteen identical nodes (representing $70 \%$ of the computational power of this cluster) were dedicated to the experimental evaluation presented in this work. All parallel computing nodes use CentOS Linux release 7.5.1804 as operating system. Slurm 17.02.7 is configured to be the batch system of the cluster. The Intel MPI version 18.0.3 was used to compile and the O3 compilation flag was enabled. 

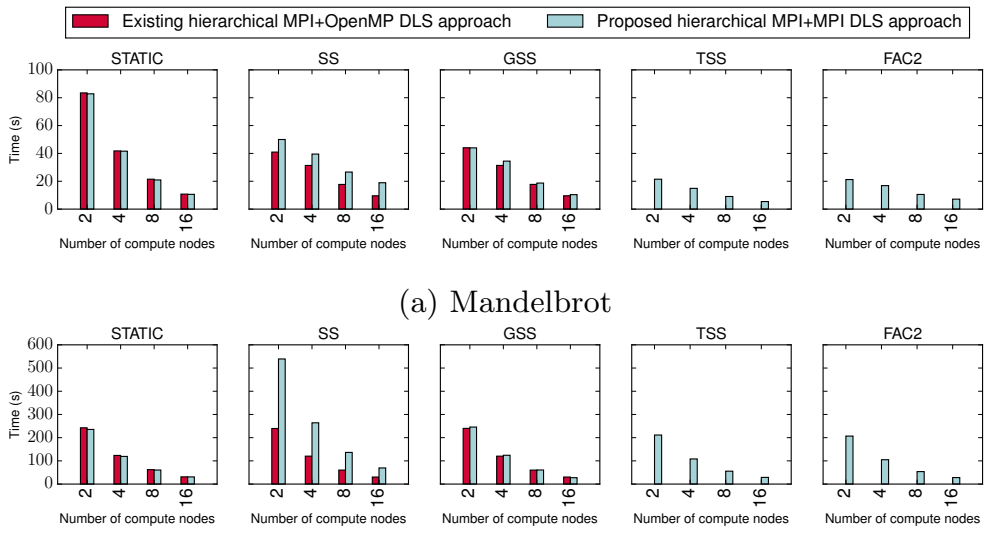

a) Mandelbrot

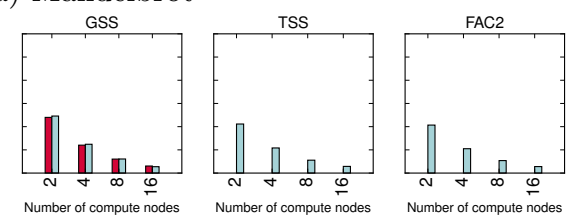

(b) PSIA

Figure 4: Parallel execution time of the main loop of both applications, Mandelbrot and PSIA. For the MPI+OpenMP approach, each worker is an OpenMP thread, and the total MPI processes per one compute node is one process. For the MPI+MPI approach, each worker is an MPI process, and the total MPI processes per one compute node is 16 processes. STATIC is the first level of scheduling (the inter-node scheduling).

\section{Experimental Evaluation}

The OpenMP standard currently supports three loop scheduling techniques: static, dynamic, and guided (cf. Table 1). As discussed in Section 2, more loop scheduling techniques were implemented in an OpenMP runtime library, called LaPeSD-libGOMP 31]. However, for accurate performance measurements, we wanted to use the most optimized software installed on miniHPC. Given that miniHPC (the target system) is an Intel-based cluster, the Intel software stack was selected, and therefore, scheduling experiments that have TSS and FAC at the shared-memory level were only performed using the proposed MPI+MPI approach. The use of LaPeSD-libGOMP, instead of Intel OpenMP runtime library, enables more DLS techniques, and it is planned as future work.

In this section, the notation of $\mathrm{X}+\mathrm{Y}$ is used to represent scheduling combinations, where $\mathrm{X}$ is a DLS technique used at the inter-node level and $\mathrm{Y}$ is a DLS technique used to at the intra-node level. $\mathrm{X}$ and $\mathrm{Y}$ can refer to one DLS technique.

Figures 4 to 7 show the performance of executing Mandelbrot and PSIA with two levels of DLS techniques. Figure 4 shows the first combination of DLS techniques where STATIC is used to schedule the workload across multiple compute nodes. An important observation is that when SS is selected to schedule the workload within one computing node, the proposed MPI+MPI approach has the poorest performance compared to the MPI+OpenMP. The reason is due to 
the use of MPI_Win_lock and MPI_Win_sync. These functions provide an exclusive access to the local work queue (see Figure 1), and consequently, maintain the work queue. The MPI_Win_lock uses a lock polling technique where an MPI process repeatedly issues lock-attempt messages until the lock is granted [38. Consequently, the number of lock-attempt messages increases when multiple processes try to acquire the same lock at the same time, and more overhead is introduced.

Another observation is that all hierarchical DLS techniques, except SS, implemented with the proposed MPI+MPI approach have the same performance compared to their counterparts implemented using the MPI+OpenMP approach. The reason is that using STATIC at the inter-node level means there is only one scheduling round at that level. However, achieving the same results also indicates that the proposed approach did not introduce a significant overhead to the DLS techniques.

Figure 5 shows the second combination of DLS techniques where GSS is used to schedule the workload across the compute nodes. For both applications, the proposed MPI+MPI approach outperformed the MPI+OpenMP. The results of the GSS+STATIC combination shows the advantage of the proposed approach, where avoiding the unnecessary synchronization between the workers (OpenMP threads) has a significant adverse impact. For instance, in Mandelbrot and using the proposed approach, the parallel execution times of the GSS+STATIC combination were 19.6 and 3.1 seconds on the smallest and the largest sys-

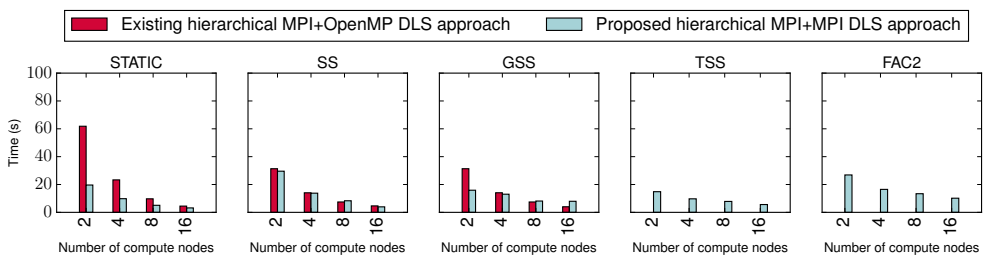

(a) Mandelbrot
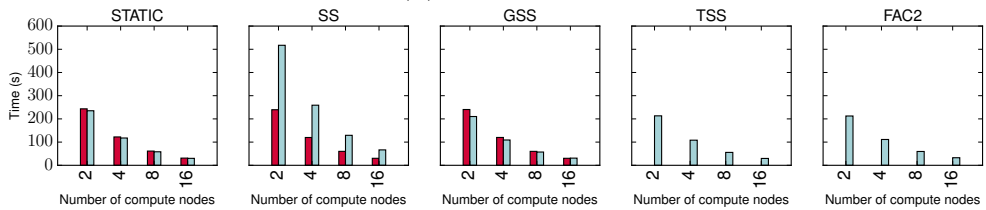

(b) PSIA

Figure 5: Parallel execution time of the main loop of both applications, Mandelbrot and PSIA. For the MPI+OpenMP approach, each worker is an OpenMP thread, and the total MPI processes per one compute node is one process. For the MPI+MPI approach, each worker is an MPI process, and the total MPI processes per one compute node is 16 processes. GSS is the first level of scheduling (the inter-node scheduling). 
tem sizes, respectively. The same scheduling combination GSS+STATIC using $\mathrm{MPI}+$ OpenMP required 61.5 and 4.5 seconds on the smallest and the largest system sizes, respectively. In PSIA, the performance trend was repeated, i.e., the GSS+STATIC using the proposed MPI+MPI approach outperformed its counterpart implemented using the MPI+OpenMP approach. For instance, on the smallest systems size, the parallel execution times were 233 and 245 seconds using the proposed MPI+MPI approach and the MPI+OpenMP approach, respectively. However, the two approaches had the same performance when executing on the largest system size. The reason is the decreased load imbalance in PSIA compared to that in Mandelbrot. For the GSS+GSS combination, the DLS techniques implemented using the proposed MPI+MPI approach also outperformed their counterparts implemented using the MPI+OpenMP approach.

As discussed in earlier in this Section, we decided to use the Intel software stack. Therefore, it was not possible to perform the remaining combinations: GSS+TSS and GSS+FAC2 using MPI+OpenMP, i.e., the Intel OpenMP runtime library only supports the following loop scheduling techniques: static, dynamic and guided.

Figures 6 and 7 show the third and the fourth combinations of DLS techniques where TSS and FAC2 are used to schedule the workload across multiple compute nodes, respectively. Similar to Figure 5, the proposed approach significantly outperformed the MPI+OpenMP approach when STATIC is selected for scheduling the computational workload within one compute node. For the rest

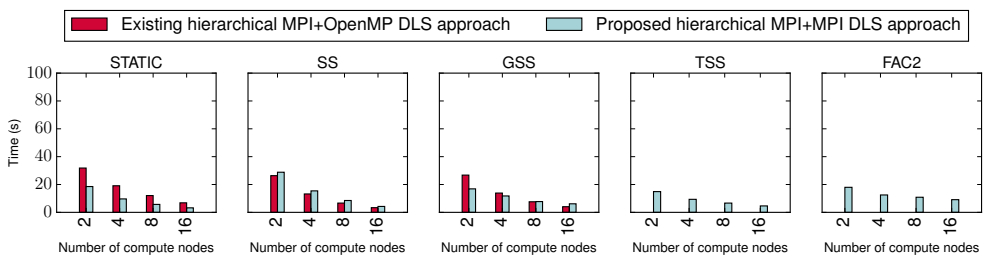

(a) Mandelbrot
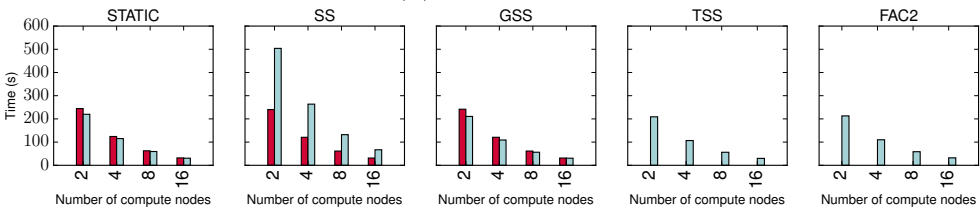

(b) PSIA

Figure 6: Parallel execution time of the main loop of both applications, Mandelbrot and PSIA. For the MPI+OpenMP approach, each worker is an OpenMP thread, and the total MPI processes per one compute node is one process. For the MPI+MPI approach, each worker is an MPI process, and the total MPI processes per one compute node is 16 processes. TSS is the first level of scheduling (the inter-node scheduling). 

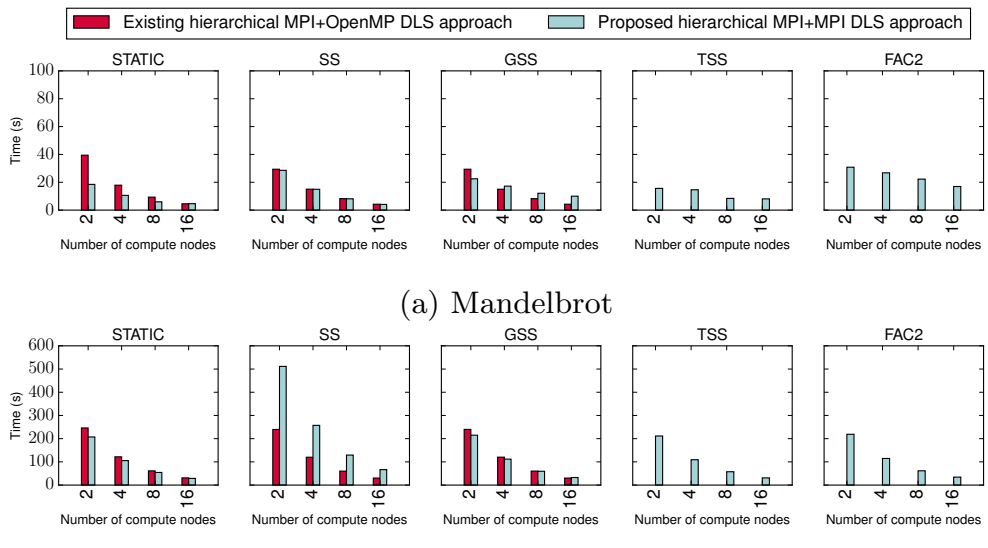

(b) PSIA

Figure 7: Parallel execution time of the main loop of both applications, Mandelbrot and PSIA. For the MPI+OpenMP approach, each worker is an OpenMP thread, and the total MPI processes per one compute node is one process. For the MPI+MPI approach, each worker is an MPI process, and the total MPI processes per one compute node is 16 processes. FAC2 is the first level of scheduling (the inter-node scheduling).

of the scheduling combinations, both approaches have the same performance. The only exception is when applying SS at the shared-memory level. The proposed approach has the worst performance compared to the MPI+OpenMP approach. The reason is that SS achieves the maximum load balance, and most of the workers (OpenMP threads) finishes at the same time. This scenario will avoid the long synchronization time before getting new chunks.

The last observation is related to the performance of the PSIA when applying any combination that has the SS using the proposed approach. PSIA has less load imbalance than Mandelbrot and the proposed approach has already a significant overhead when employing SS, and consequently, the adverse impact of the large associated scheduling overhead of SS is more visible in PSIA than Mandelbrot. 


\section{Conclusion and Future Work}

The implementation of hierarchical dynamic loop self-scheduling techniques is essential to enable scalable application performance. When STATIC is used for the intra-node level scheduling, the implementation that uses MPI+MPI approach outperformed the one that uses hybrid MPI+OpenMP approach. This highlights and confirms the capability of the MPI+MPI approach to eliminate the unrequired synchronization at the intra-node level. On the contrary, the MPI+MPI approach shows significant limited performance when many MPI processes on the same shared-memory system try to simultaneously access the local work queue. The important observation of the present work is that the scheduling overhead associated with using MPI shared-memory to implement DLS techniques is higher than OpenMP. Therefore, the use of the MPI+MPI approach is only recommended for developing hierarchical DLS techniques when its associated overhead is less than the synchronization overhead associated with the use of OpenMP. The assessment of the performance and the development efforts associated with the use of nowait clause compared to the use of the proposed MPI+MPI hierarchical DLS approach are planned as future work. Moreover, a detailed evaluation of the synchronization overhead between the OpenMP threads with and without the use of nowait clause is needed and planned as future work.

\section{Acknowledgment}

This work has been supported by the Swiss National Science Foundation in the context of the Multi-level Scheduling in Large Scale High Performance Computers (MLS) grant number 169123 and by the Swiss Platform for Advanced Scientific Computing (PASC) project SPH-EXA: Optimizing Smooth Particle Hydrodynamics for Exascale Computing. 


\section{References}

[1] MPI Forum, "Message-Passing Interface," https://www.mpi-forum.org, [Online; accessed 16 May 2018].

[2] T. Hoefler, J. Dinan, D. Buntinas, P. Balaji, B. Barrett, R. Brightwell, W. Gropp, V. Kale, and R. Thakur, "MPI + MPI: a new hybrid approach to parallel programming with MPI plus shared memory," Computing, vol. 95, no. 12 , pp. 1121-1136, 2013.

[3] T. Hoefler, J. Dinan, D. Buntinas, P. Balaji, B. W. Barrett, R. Brightwell, W. Gropp, V. Kale, and R. Thakur, "Leveraging MPIs one-sided communication interface for shared-memory programming," in European MPI Users' Group Meeting, 2012, pp. 132-141.

[4] OpenMP Architecture Review Board, "OpenMP Application Programming Interface," https://www.openmp.org/wp-content/uploads/ OpenMP-API-Specification-5.0.pdf, 2018, [Online; accessed 25 January 2019].

[5] H. Jin, D. Jespersen, P. Mehrotra, R. Biswas, L. Huang, and B. Chapman, "High performance computing using MPI and OpenMP on multi-core parallel systems," Parallel Computing, vol. 37, no. 9, pp. 562-575, 2011.

[6] L. Smith and M. Bull, "Development of mixed mode MPI/OpenMP applications," Scientific Programming, vol. 9, no. 2-3, pp. 83-98, 2001.

[7] M. J. Chorley and D. W. Walker, "Performance analysis of a hybrid MPI/OpenMP application on multi-core clusters," Journal of Computational Science, vol. 1, no. 3, pp. 168-174, 2010.

[8] I. Banicescu, V. Velusamy, and J. Devaprasad, "On the scalability of dynamic scheduling scientific applications with adaptive weighted factoring," Journal of Cluster Computing, vol. 6, no. 3, pp. 215-226, 2003.

[9] L. DeRose, B. Homer, and D. Johnson, "Detecting application load imbalance on high end massively parallel systems," in European Conference on Parallel Processing, 2007, pp. 150-159.

[10] R. L. Cariño and I. Banicescu, "A load balancing tool for distributed parallel loops," Journal of Cluster Computing, vol. 8, no. 4, pp. 313-321, 2005.

[11] I. Banicescu, R. L. Cariño, J. P. Pabico, and M. Balasubramaniam, "Design and implementation of a novel dynamic load balancing library for cluster computing," Journal of Parallel Computing, vol. 31, no. 7, pp. 736-756, 2005 .

[12] R. L. Cariño, I. Banicescu, T. Rauber, and G. Rünger, "Dynamic loop scheduling with processor groups," in Proceedings of the 17th International Conference on Parallel and Distributed Computing Systems., 2004, pp. 7884. 
[13] A. T. Chronopoulos, S. Penmatsa, N. Yu, and D. Yu, "Scalable loop selfscheduling schemes for heterogeneous clusters," International Journal of Computational Science and Engineering, vol. 1, no. 2-4, pp. 110-117, 2005.

[14] C.-C. Wu, C.-T. Yang, K.-C. Lai, and P.-H. Chiu, "Designing parallel loop self-scheduling schemes using the hybrid MPI and OpenMP programming model for multi-core Grid systems," The Journal of Supercomputing, vol. 59, no. 1, pp. 42-60, 2012.

[15] A. Eleliemy and F. M. Ciorba, "Dynamic Loop Scheduling Using MPI Passive-Target Remote Memory Access," in Proceedings of the 27th Euromicro International Conference on Parallel, Distributed and Networkbased Processing, Feb 2019.

[16] M. Effatparvar and M. Garshasbi, "A genetic algorithm for static load balancing in parallel heterogeneous systems," Procedia-Social and Behavioral Sciences, vol. 129, pp. 358-364, 2014.

[17] R. Biswas, S. K. Das, D. J. Harvey, and L. Oliker, "Parallel dynamic load balancing strategies for adaptive irregular applications," Applied Mathematical Modelling, vol. 25, no. 2, pp. 109-122, 2000.

[18] Y. Li, Y. Yang, M. Ma, and L. Zhou, "A hybrid load balancing strategy of sequential tasks for grid computing environments," Future Generation Computer Systems, vol. 25, no. 8, pp. 819-828, 2009.

[19] M. Garcia, J. Corbalan, and J. Labarta, "LeWI: A runtime balancing algorithm for nested parallelism," in Proceedings of the International Conference on Parallel Processing (ICPP), 2009, pp. 526-533.

[20] M. Garcia, J. Corbalan, R. M. Badia, and J. Labarta, "A dynamic load balancing approach with SMPSuperscalar and MPI," in Facing the MulticoreChallenge II, 2012, pp. 10-23.

[21] T. Rauber and G. Rünger, "Tliba library to support programming with hierarchical multi-processor tasks," Journal of Parallel and Distributed Computing, vol. 65, no. 3, pp. 347-360, 2005.

[22] Z. Fang, P. Tang, P.-C. Yew, and C.-Q. Zhu, "Dynamic processor selfscheduling for general parallel nested loops," IEEE Transactions on Computers, vol. 39, no. 7, pp. 919-929, 1990.

[23] C. D. Polychronopoulos and D. J. Kuck, "Guided Self-Scheduling: A Practical Scheduling Scheme for Parallel Supercomputers," IEEE Transactions on Computers, vol. 100, no. 12, pp. 1425-1439, 1987.

[24] T. H. Tzen and L. M. Ni, "Trapezoid Self-Scheduling: A Practical Scheduling Scheme for Parallel Compilers," IEEE Transactions on parallel and distributed systems, vol. 4, no. 1, pp. 87-98, 1993. 
[25] S. Flynn Hummel, E. Schonberg, and L. E. Flynn, "Factoring: A method for scheduling parallel loops," Communications of the ACM, vol. 35, no. 8, pp. 90-101, 1992.

[26] S. Flynn Hummel, J. Schmidt, R. Uma, and J. Wein, "Load-sharing in heterogeneous systems via weighted factoring," in Proceedings of the 8th annual ACM symposium on Parallel algorithms and architectures, 1996, pp. $318-328$.

[27] A. T. Chronopoulos, R. Andonie, M. Benche, and D. Grosu, "A class of loop self-scheduling for heterogeneous clusters," in Proceedings of International Conference on Cluster Computing, 2001, pp. 282-291.

[28] T. Peiyi and Y. Pen-Chung, "Processor self-scheduling for multiple-nested parallel loops," in Proceedings of the International Conference on Parallel Processing, August 1986, pp. 528-535.

[29] R. L. Cariño and I. Banicescu, "Dynamic load balancing with adaptive factoring methods in scientific applications," The Journal of Supercomputing, vol. 44, no. 1, pp. 41-63, 2008.

[30] W.-C. Shih, C.-T. Yang, and S.-S. Tseng, "A performance-based parallel loop scheduling on grid environments," The Journal of Supercomputing, vol. 41, no. 3, pp. 247-267, 2007.

[31] F. M. Ciorba, C. Iwainsky, and P. Buder, "OpenMP loop scheduling revisited: making a case for more schedules," in Proceedings of the 2018 International Workshop on OpenMP (iWomp 2018), September 2018.

[32] A. Eleliemy, M. Fayze, R. Mehmood, I. Katib, and N. Aljohani, "Loadbalancing on Parallel Heterogeneous Architectures: Spin-image Algorithm on CPU and MIC," in Proceedings of the 9th EUROSIM Congress on Modelling and Simulation, September 2016, pp. 623-628.

[33] A. Eleliemy, D. Hegazy, and W. S. Elkilani, "MPI parallel implementation of 3D object categorization using spin-images," in 2013 9th International Computer Engineering Conference (ICENCO), 2013, pp. 25-31.

[34] B. B. Mandelbrot, "Fractal aspects of the iteration of z $\Lambda \mathrm{z}(1-\mathrm{z})$ for complex $\Lambda$ and z," Annals of the New York Academy of Sciences, vol. 357, no. 1, pp. 249-259, 1980.

[35] A. E. Johnson, "Spin-Images: A Representation for 3-D Surface Matching," Ph.D. dissertation, Robotics Institute, Carnegie Mellon University, August 1997.

[36] A. Eleliemy, A. Mohammed, and F. M. Ciorba, "Efficient generation of parallel spin-images using dynamic loop scheduling," in Proceedings of the 8th International Workshop on Multicore and Multithreaded Architectures 
and Algorithms (M2A2 2017) in conjunction with the 19th IEEE International Conference for High Performance Computing and Communications (HPCC 2017), December 2017, p. 8.

[37] The HPC group at the University of Basel, "miniHPC," https://hpc.dmi. unibas.ch/HPC/miniHPC.html, [Online; accessed 21 May 2019].

[38] X. Zhao, P. Balaji, and W. Gropp, "Scalability challenges in current MPI one-sided implementations," in International Symposium on Parallel and Distributed Computing (ISPDC), 2016, pp. 38-47. 